\title{
CoRoT high-precision photometry of the B0.5 IV star HD 51756
}

\author{
P. I. Pápics ${ }^{1}$, M. Briquet ${ }^{1, \star \star \star \star}$, M. Auvergne ${ }^{2}$, C. Aerts ${ }^{1,3}$, P. Degroote ${ }^{1}$, E. Niemczura $^{1,4}$, M. Vučković ${ }^{1,5}$, \\ K. Smolders ${ }^{1 \dagger}$, E. Poretti ${ }^{8}$, M. Rainer ${ }^{8}$, M. Hareter ${ }^{9}$, A. Baglin ${ }^{10}$, F. Baudin ${ }^{6}$, C. Catala ${ }^{2}$, E. Michel $^{2}$, and R. Samadi ${ }^{2}$ \\ 1 Instituut voor Sterrenkunde, K.U. Leuven, Celestijnenlaan 200D, 3001 Leuven, Belgium \\ e-mail: peter.papics@ster.kuleuven.be \\ 2 LESIA, UMR8109, Université Pierre et Marie Curie, Université Denis Diderot, Observatoire de Paris, 92195 Meudon Cedex, \\ France \\ 3 Department of Astrophysics, IMAPP, University of Nijmegen, PO Box 9010, 6500 GL Nijmegen, The Netherlands \\ ${ }^{4}$ Instytut Astronomiczny, Uniwersytet Wroclawski, Kopernika 11, 51-622 Wroclaw, Poland \\ 5 European Southern Observatory (ESO), Alonso de Córdova 3107, Vitacura, Cassilla 19001, Santiago, Chile \\ ${ }^{6}$ INAF - Osservatorio Astronomico di Brera, via E. Bianchi 46, 23807 Merate (LC), Italy \\ 7 Institut für Astronomie, Universität Wien, Türkenschanzstrasse 17, 1180 Vienna, Austria \\ ${ }^{8}$ Laboratoire AIM, CEA/DSM-CNRS-Université Paris Diderot, CEA, IRFU, SAp, Centre de Saclay, 91191 Gif-sur-Yvette, France \\ 9 Institut d'Astrophysique Spatiale, CNRS/Univ. Paris-Sud, Bât. 121, 91405 Orsay Cedex, France
}

Received 11 November 2010 / Accepted 14 January 2011

\begin{abstract}
Context. OB stars are important constituents for the ecology of the Universe, and there are only a few studies on their pulsational properties detailed enough to provide important feedback on current evolutionary models.

Aims. Our goal is to analyse and interpret the behaviour present in the CoRoT light curve of the B0.5 IV star HD 51756 observed during the second long run of the space mission and to determine the fundamental stellar parameters from ground-based spectroscopy gathered with the CORALIE and HARPS instruments after checking for signs of variability and binarity, thus making a step further in mapping the top of the $\beta$ Cep instability strip.

Methods. We compared the newly obtained high-resolution spectra with synthetic spectra of late O-type and early B-type stars computed on a grid of stellar parameters. We matched the results with evolutionary tracks to estimate stellar parameters. We used various time series analysis tools to explore the nature of the variations present in the light curve. Additional calculations were carried out based on distance and historical position measurements of the components to impose constraints on the binary orbit.

Results. We find that HD 51756 is a wide binary with both a slow $\left(v \sin i \approx 28 \mathrm{~km} \mathrm{~s}^{-1}\right)$ and a fast $\left(v \sin i \approx 170 \mathrm{~km} \mathrm{~s}^{-1}\right)$ early-B rotator whose atmospheric parameters are similar $\left(T_{\text {eff }} \approx 30000 \mathrm{~K}\right.$ and $\left.\log g \approx 3.75\right)$. We are unable to detect pulsation in any of the components, and we interpret the harmonic structure in the frequency spectrum as a sign of rotational modulation, which is compatible with the observed and deduced stellar parameters of both components.

Conclusions. The non-detection of pulsation modes provides a feedback on the theoretical treatment, given that non-adiabatic computations applied to appropriate stellar models predict the excitation of both pressure and gravity modes for the fundamental parameters of this star.
\end{abstract}

Key words. stars: variables: general - stars: early-type - stars: oscillations - stars: individual: HD 51756 - stars: rotation - binaries: spectroscopic

\section{Introduction}

One of the major observing programmes for the asteroseismology channel of the CoRoT satellite (Baglin et al. 2006) is to derive details of the internal physics of stars within the instability

* The CoRoT space mission was developed and is operated by the French space agency CNES, with participation of ESA's RSSD and Science Programmes, Austria, Belgium, Brazil, Germany, and Spain.

$\star \star$ Based on data gathered with Coralie installed on the $1.2 \mathrm{~m}$ Euler telescope at La Silla, Chile; and HARPs installed on the $3.6 \mathrm{~m}$ ESO telescope (ESO Large Programme 182.D-0356) at La Silla, Chile.

$\star \star \star$ Appendix A is only available in electronic form at http: //www . aanda.org

$\star \star \star \star$ Postdoctoral Fellow of the Fund for Scientific Research, Flanders.

Aspiring Fellow of the Fund for Scientific Research, Flanders. strip of early-type B pulsators (e.g., Michel et al. 2006). A major motivation of this programme was to understand the excitation problems reported for some of the observed modes in several prototypical, bright $\beta$ Cep stars by groups using independent stellar evolution and pulsation codes (e.g., Pamyatnykh et al. 2004; Ausseloos et al. 2004; Handler et al. 2006; Desmet et al. 2009), while other class members show oscillations as predicted by theory (Aerts et al. 2003; Mazumdar et al. 2006; Briquet et al. 2007b; Handler 2009). The goal was also to study stars of spectral type $\mathrm{O}$ or $\mathrm{B} 0$, which are higher up in the predicted $\beta \mathrm{Cep}$ strip than most class members observed from the ground prior to CoRoT, to see if the emptiness of that part of the strip is removed when going from mmag to $\mu$ mag precision in photometric data. A third motivation was to obtain data of higher precision than from the ground and with no interruption, in order to 
establish once and for all the firm detection of non-radial gravity modes seemingly present in the ground-based spectroscopy of rapidly rotating Be stars (e.g., Rivinius et al. 2003), as well as to interpret those in terms of oscillations of rapid rotators. A number of early-type B stars were thus selected to fulfil these goals, keeping in mind the strong pointing restrictions of the satellite, as well as the brightness limitations (e.g., Auvergne et al. 2009). The star HD 51756 (B0.5 IV) studied in this paper was selected as the target in this context, as were $6 \mathrm{O}$ stars measured during a short run. All further planned B targets have later spectral type due to the pointing restrictions of the satellite.

The slowly rotating B stars observed by CoRoT's asteroseismology CCDs so far have led to some remarkable results. The only accessible, known $\beta$ Cep star, HD 180642 (B1.5 II-III), turned out to have numerous self-excited modes rather than one dominant radial mode as was thought prior to CoRoT (Degroote et al. 2009; Briquet et al. 2009), and many of the observed modes are resonantly coupled. Moreover, the star was found to have modes of a stochastic nature as well (Belkacem et al. 2009), which was never found before in a $\beta$ Cep pulsator and which requires the presence of a surface convection zone capable of exciting such modes. Stochastically excited modes exhibiting a constant frequency spacing were, surprisingly, detected in the O8.5 V star HD 46149 (Degroote et al. 2010b). On the other hand, the O9V star HD 46202 turns out to be the most massive star known to date with $\beta$ Cep-like pulsation frequencies, but for which present excitation computations fail to predict the observed oscillations (Briquet et al. 2011). Furthermore, at the cool border of the $\beta$ Cep instability strip, the B3 V star HD 50230 revealed oscillations of hybrid nature, i.e., the presence of both gravity modes as expected in slowly pulsating B stars and pressure modes as in the classical $\beta$ Cep stars. The gravity modes of this star displayed a period spacing and a periodic deviation thereof, which allowed one to prove the existence of a chemically inhomogeneous zone around the fully mixed core, which has not yet been taken into account in current B star models (Degroote et al. 2010a).

As for the rapidly rotating early-Be stars, HD 49330 (B0.5 IVe) turned out to be a clear case where multiple mode beating was observed in real time, and this phenomenon was found to be connected with an outburst feeding the circumstellar disk (Huat et al. 2009; Floquet et al. 2009). This supports the early findings of Baade et al. (1988) and Rivinius et al. (2001) that the outbursts detected in the B2e star $\mu$ Cen have a pulsational origin and are connected to multimode beating. This outburst phenomenon has so far not been found for the other Be stars that were monitored intensively by CoRoT, but those targets are of later spectral type (Neiner et al. 2009, HD 181231, B5 IVe; Gutiérrez-Soto et al. 2009, HD 175869, B8 IIIe). There is still no acceptable seismic modelling of the interior structure of a Be star, owing to the mathematical complexity of treating stellar oscillations in a deformed star.

It is obvious from these recent findings that the diversity of stellar oscillations in and near the $\beta$ Cep instability strip is much more than anticipated before the CoRoT mission. This must imply that details in the internal physics of the various stars, such as their internal rotation, mixing, settling, and radiative levitation due to atomic diffusion, etc., may be different. Some problems may also be solved by increasing the opacity in the excitation layers. In an attempt to increase the number of well-studied early-B stars, HD 51756 (B0.5 IV) was monitored by CoRoT's seismology CCDs and studied in parallel with ground-based spectroscopy.

\section{Fundamental parameters}

\subsection{Prior to our study}

HD 51756 - a bright $\left(V_{\text {mag }}=7.23\right)$ B0.5 IV field-star in the constellation of Monoceros - was first listed among early-type high-luminosity objects by Münch (1951), where it was classified as a B3 star from objective-prism plates. The luminosity class and a more precise spectral type of B0.5 IV was determined by Morgan et al. (1955), and has generally been used since then. Although there are exceptions - a B0 III classification by Dworetsky et al. (1982), a B1 V by Abt (2008), and B1/2 Ib by Wright et al. (2003) - there seems to be a quite good agreement on the spectral type, but not the luminosity class.

Measurements by Burnham (1875) showed that HD 51756 is a triple system (catalogued as BU 327, but widely known as WDS J06585-0301), consisting of two almost equally bright ( $\Delta m \approx 0.3 \mathrm{mag}$ ) close components with a separation of $\rho \approx 0.7^{\prime \prime}$ and a fainter star further away $\left(\Delta m \approx 4 \mathrm{mag}, \rho \approx 13.7^{\prime \prime}\right)$. Unfortunately, only one historical radial velocity (RV) measurement $\left(v_{\mathrm{rad}}=25 \pm 5 \mathrm{~km} \mathrm{~s}^{-1}\right)$ can be found (Wilson 1953). Searching for $\beta$ Cep stars, Hill (1967) took the first photometric time-series and concluded that this field star was constant. Among other parameters, he also measured the projected rotational velocity to be $v \sin i=30 \mathrm{~km} \mathrm{~s}^{-1}$. There is another measurement from Glebocki \& Stawikowski (2000) claiming $v \sin i=40 \mathrm{~km} \mathrm{~s}^{-1}$.

More information we found concerns $V_{\mathrm{mag}}=7.23, \beta_{\mathrm{mag}}=$ $2.569, M_{\mathrm{v}}=-4.1$, the colours of $(B-V)=-0.10$ and $(U-B)=$ -0.93 , and the colour excess of $E(B-V)=0.19$. In addition to these, $\left(U V^{1}-V\right)=-2.60$ and $E(U V)=1.07$ were measured by Weber et al. (1971). A distance modulus of $V_{0}-M_{\mathrm{v}}=10.7$ is given by Vogt (1976), which leads to a distance of $1.38 \mathrm{kpc}$ (1.04 kpc with extinction taken into account). There are similar measurements of $1.3 \pm 0.5 \mathrm{kpc}$ (Avedisova \& Kondratenko 1984, for the BFS $55 \mathrm{H}$ in region ${ }^{2}$, which is claimed to be ionised by the nearby multiple subgiant, HD 51756), $1.6 \mathrm{kpc}$ (Savage et al. 1985) and $2.156_{-0.443}^{+0.559} \mathrm{kpc}$ (Kaltcheva \& Hilditch 2000, with $M_{\mathrm{v}}=-5.075$ from $u v b y \beta$ photometry). The original Hipparcos (Perryman et al. 1997) parallax is $0.56 \pm 4.8$ mas, while the value from the new reduction (van Leeuwen 2007) is $-1.94 \pm 1.13$ mas. Unfortunately, none of these has lead to a useful distance estimate. Empirical temperature calibrations (Gulati et al. 1989) gave $\log T_{\text {eff }}=4.522 \pm 0.051 \mathrm{~K}$ from QUV, $\log T_{\text {eff }}=4.532 \pm$ $0.042 \mathrm{~K}$ from $(m 1965-V)_{0}$, and $\log T_{\text {eff }}=4.530 \pm 0.045 \mathrm{~K}$ from $(B-V)_{0}$ values, corresponding to - with errors taken into account - an effective temperature range of 29500-37 $500 \mathrm{~K}$.

\subsection{New spectroscopy}

In the framework of ground-based preparatory and follow-up observations for the CoRoT space mission, high-resolution and medium-to-high signal-to-noise spectra were taken with the Coralie (Baranne et al. 1996; Queloz et al. 2001, $R \approx 50000$ ) and Harps (Mayor et al. 2003, $R \approx 80000$ ) instruments (78 and 15 measurements, respectively). See Table 1 for a summary of the spectroscopic observations, listing the number of observed spectra, the heliocentric Julian dates (HJD), the signal-to-noise ratios, typical exposure times, and the resolution of the spectrograph for each observing run. In addition to these, one spectrum

\footnotetext{
1 The $U V$ filter is centred at $1500 \AA$.

2 For the same region Fich \& Blitz (1984) found a kinematic distance of $1.89 \pm 0.73 \mathrm{kpc}$ determined by using $\mathrm{CO}$ velocities of the associated molecular clouds and the $\mathrm{CO}$ rotation curve of the outer galaxy.
} 
Table 1. Logbook of the new spectroscopic observations of HD 51756 obtained between January 2009 and December 2009 - grouped by observing runs.

\begin{tabular}{lccccccc}
\hline \hline Instrument & $N$ & HJD begin & HJD end & $\langle S N R\rangle^{a}$ & $S N R$-range ${ }^{a}$ & $T_{\exp }(\mathrm{s})$ & $R$ \\
\hline CoRALIE & 53 & 2454842 & 2454852 & 124 & {$[97,142]$} & 1500 & 50000 \\
CoRALIE & 25 & 2454912 & 2454922 & 130 & {$[99,160]$} & {$[1500,2000]$} & 50000 \\
HARPS & 15 & 2455174 & 2455195 & 293 & {$[265,330]$} & {$[450,840]$} & 80000 \\
\hline
\end{tabular}

Notes. ${ }^{(a)}$ The signal-to-noise ratios were calculated in the line free region of [5370, $\left.5400 \AA\right]$.
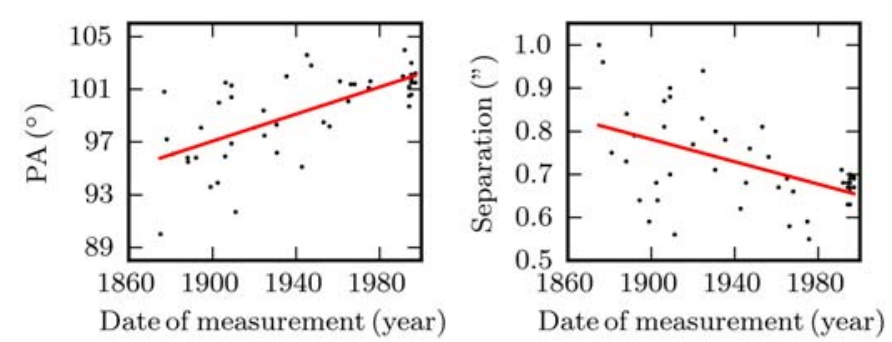

Fig. 1. Position angle (left) and separation (right) measurements of the two bright and close components of HD 51756 (WDS J06585-0301 AB) from The Washington Double Star Catalog, with a linear trend fitted to them.

gathered with Feros (Kaufer et al. $1999, R \approx 48000$ ) was taken from the GAUDI archive (Solano et al. 2005).

We performed a careful normalisation of each spectrum, using cubic splines which were fitted through some tens of points at fixed wavelengths, where the continuum was known to be free of spectral lines and telluric features. The final selection of these nodal points was made after several tests and quality checks, making sure that, e.g., the wings of the Balmer-lines are not affected, but all artificial features (like periodic waves in the continuum) are corrected in the process. Cosmic removal via pixel-by-pixel sigma clipping and a sophisticated order merging (taking into account the signal-to-noise values in the overlapping ranges and correcting for the sometimes slightly different flux levels of the overlapping orders) were also done by the same semi-automatic script. This is much faster and less subjective than one-by-one manual normalisation.

Radial velocities were measured from cross-correlated profiles - calculated from the three strongest Si III lines (at $4553 \AA$, $4568 \AA$, and $4575 \AA$ ) using the least-square deconvolution technique of Donati et al. (1997) - by fitting a simple Gaussian to them. It is clear from the determined radial velocities, that the orbital period has a much longer timescale than the timespan of the spectroscopic observations, and the orbit cannot be fitted on this number of measurements. The idea of a very long orbital period is confirmed by position measurements covering 122 years found in the Washington Double Star Catalog also (see Table A.1), which show only a minor change during more than a century in position angle (which slowly increases) and in separation (which slowly decreases); see Fig. 1.

By visual inspection of the averaged spectrum of both instruments (and the original spectra), we conclude that HD 51756 is a double-line spectroscopic binary (SB2) with a slow (referred to as the "primary" from now on, as the sharp spectral features are connected to this star) and a fast rotator (secondary) star (see Fig. 2). Seeing both components in the spectrum is not surprising because the separation of the components is only $0.7^{\prime \prime}$, which is smaller than the fiber aperture of CoRALIE ( $\left.2^{\prime \prime}\right)$ and Harps ( $\left(1^{\prime \prime}\right)$. It is also below the average seeing of La Silla $\left(0.9^{\prime \prime}\right)$, while the difference in brightness of the primary and the secondary is small.
Knowing the RV values corresponding to each exposure, we transferred all spectra to the laboratory rest frame (of the primary). At this point, we re-ran the normalisation script (this way the nodal points are really at the same rest wavelength) and calculated the average spectrum for both instruments, using weights according to the calculated or given $S N R$ values of each spectrum. The two average spectra calculated from CORALIE and HARPS are almost perfectly identical (with an SNR [5370-5400 $\AA$ ] of 873 and 1016, respectively). The spectra only show absorption features, except for the Fe III emission line at $5243 \AA$.

Though in the case of the individual Coralie spectra, the $S N R$ levels were a bit lower than is typical for such analysis, we searched for line-profile variations using the FAMIAS software package (Zima 2008), but without any positive detection.

\subsection{Fundamental parameters}

An SB2 binary is extremely useful when the orbit can be fitted and, after spectral disentangling, a full analysis can be carried out on both components separately. In our case, this is not possible, owing to the lack of knowledge concerning the orbital parameters.

To have a relatively fast but still accurate solution, we decided to carry out a full grid search with seven free parameters ( $T_{\text {eff }}, \log g$, and $v \sin i$ for both components, plus the metallicity $Z$, which was assumed to be the same for both stars), using the OSTAR2002 (Lanz \& Hubeny 2003) and BSTAR2006 (Lanz \& Hubeny 2007) grids. These sets of synthetic spectra were both calculated from NLTE, plane-parallel, hydrostatic model atmospheres, with a microturbulent velocity of $10 \mathrm{~km} \mathrm{~s}^{-1}$ for the OSTAR2002 and and $2 \mathrm{~km} \mathrm{~s}^{-1}$ for the BSTAR2006 grid. Equivalent widths of the instrumental line profiles were also taken into account while calculating the broadening of the synthetic composite spectra. Chi-square $\left(\chi^{2}\right)$ values were calculated using only regions of selected $\mathrm{HI}, \mathrm{He}$, Si III, and Si Iv lines. Some example fits are shown in Fig. 2. The effect of the fast rotator companion is clearly visible in the He I line at $4713 \AA$ (lower left panel). We had difficulty in fitting the metal lines equally well with any given setting. Some regions suggest higher (e.g., the Si III lines at $4553 \AA, 4568 \AA$, and $4575 \AA$ (lower middle)), while some others suggest lower metallicity values (e.g., the Si IV line at $4212 \AA$ (lower right)). Errors were estimated by running the grid search using both the CoRALIE and HARPS average spectra, using all four possible combinations of the two grids for the two components, and using more or fewer line regions for the $\chi^{2}$ calculation, then finally taking the most common parameters among the combinations with the lowest $\chi^{2}$ values as the final result, while the lowest and highest parameter values close to the minima of the $\chi^{2}$ set the error bars. A summary of the results is shown in Table 2 . The two stars are very similar, the only difference being a different equatorial velocity or a different stellar inclination. The errors on the effective temperature and on the sur- 

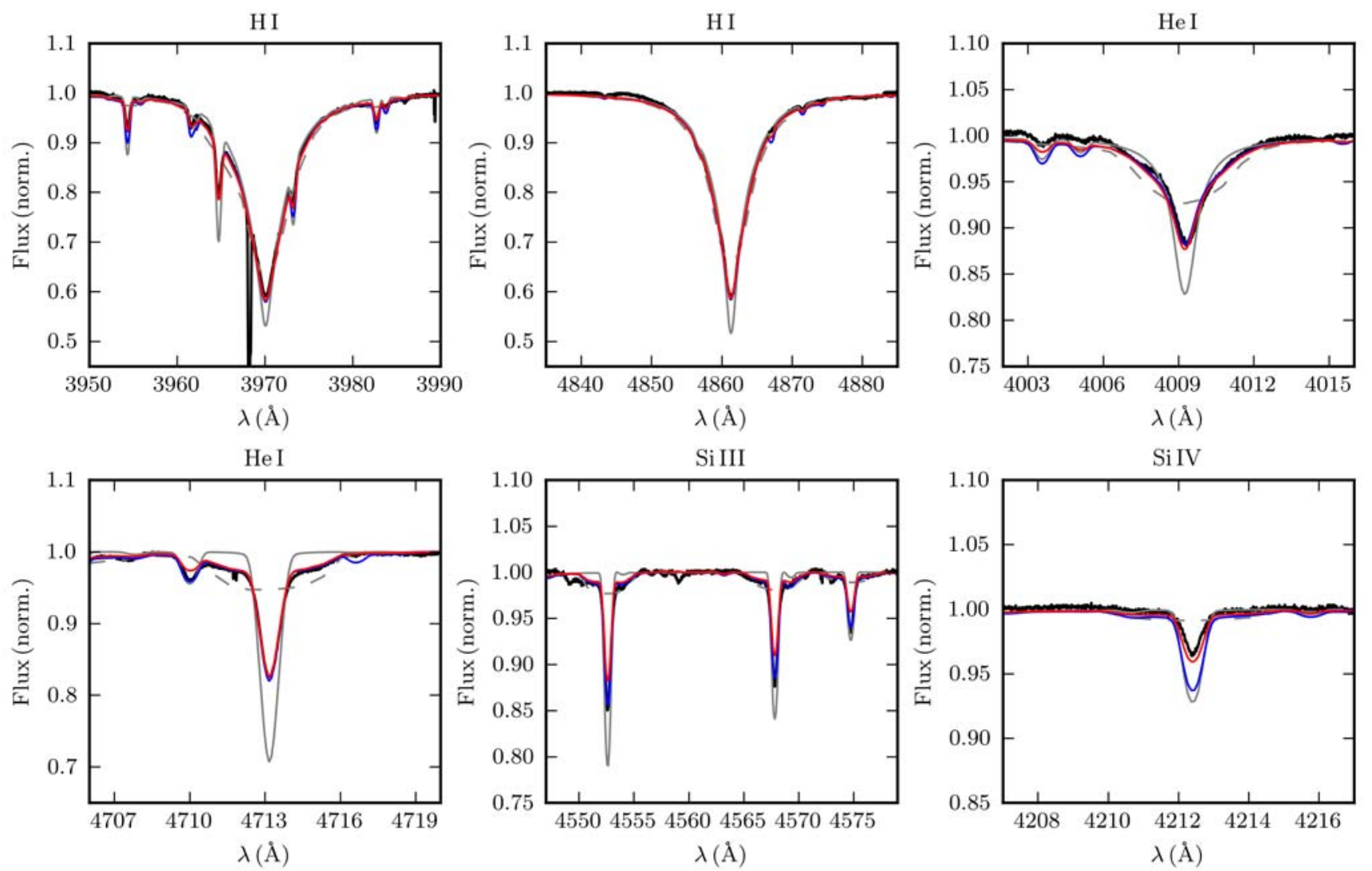

Fig. 2. Fits to the averaged Harps spectrum (black). The parameters are listed in Table 2. The fit to the primary companion is shown in solid grey, the fit to the secondary in dashed grey, and the combined fit in red. While these synthetic spectra were calculated for $Z=0.010$, we also plot the combined spectrum corresponding to $Z=0.020$ with a solid blue line to show the effect of the change in metallicity.

Table 2. Observed and corresponding model parameters.

\begin{tabular}{lcc}
\hline \hline Parameter & Primary & Secondary \\
\hline \multicolumn{3}{c}{ from spectroscopy } \\
$T_{\text {eff }}(\mathrm{K})$ & $30000 \pm 1000$ & $30000 \pm 2500$ \\
$\log g(\mathrm{cgs})$ & $3.75 \pm 0.25$ & $3.75 \pm 0.25$ \\
$v \sin i\left(\mathrm{~km} \mathrm{~s}^{-1}\right)$ & $28 \pm 4$ & $170 \pm 15$ \\
$Z$ & \multicolumn{3}{c}{$\in[0.010,0.020]$} \\
\hline \multicolumn{3}{c}{ from evolutionary tracks } \\
$\mathcal{M}\left(\mathcal{M}_{\odot}\right)$ & $18.9_{-4.4}^{+9.1}$ & $18.9_{-5.9}^{+15.1}$ \\
$R\left(R_{\odot}\right)$ & $9.6_{-3.3}^{+5.9}$ & $9.6_{-3.7}^{+7.5}$ \\
$\log L\left(L_{\odot}\right)$ & $4.8_{-0.4}^{+0.5}$ & $4.8_{-0.6}^{+0.6}$ \\
$\operatorname{age}(\mathrm{Myr})$ & $7.6_{-2.0}^{+1.1}$ & $7.6_{-3.0}^{+2.8}$ \\
\hline
\end{tabular}

face gravity might be slightly underestimated for the secondary, as this component contributes less to the overall appearance of the composite spectra because of the shallow lines caused by its high projected rotational velocity. The lowest $\chi^{2}$ values were reached by using the OSTAR2002 grid for the primary and the BSTAR2006 grid for the secondary. (Not only the microturbulent velocity, but also the atomic data included in these model atmosphere grids are slightly different.) Also, a slight trend in the full set of results suggests that the secondary might be $\approx 1000 \mathrm{~K}$ cooler than the primary, and it might have a slightly lower $\log g$ value than its companion star, but these deviations are within the given error bars. As shown below, this agrees with the observed $\Delta m=m_{2}-m_{1}=M_{2}-M_{1} \approx 0.3$ mag brightness difference of the components. From the definition of the magnitude scale and the relation between effective temperature and luminosity, we write

$\left(\frac{R_{1}}{R_{2}}\right)^{2}\left(\frac{T_{1}}{T_{2}}\right)^{4}=\frac{L_{1}}{L_{2}}=10^{\left(M_{2}-M_{1}\right) / 2.5} \approx 1.3$,

and with this expression, we can give the upper limit of the difference in effective temperature and radii of the components. Assuming the same radii, we need $T_{2}=0.937 T_{1}$ to fulfil the equation, while assuming the same temperature requires $R_{2}=$ $0.877 R_{1}$. At $T_{\text {eff }}=30000 \mathrm{~K}$ this means that the secondary can be cooler by a maximum of $\approx 2000 \mathrm{~K}$, which fits the results of the grid search within the errors.

After we determined the $T_{\text {eff }}$ and $\log g$ values of both components, we matched them with evolutionary tracks (see Figs. 3 and 4 for an overview, and Briquet et al. 2011, for a description of the input physics). As the lowest $\chi^{2}$ values indicate a minimum in between $Z=0.02$ and $Z=0.01$, we fixed the metallicity at $Z=0.014$ in accordance with Przybilla et al. (2008), who proposes the cosmic abundance standard (CAS) from early B-type stars in the solar neighbourhood. We chose $X=0.715$, also in good agreement with the proposed CAS, and set the core overshoot parameter at $\alpha_{\mathrm{ov}}=0.2$ pressure scale heights, since asteroseismic modeling results of $\beta$ Cep targets have given evidence of core overshooting of that order (Aerts et al. 2010). From the model points that lie within the error box set by the $T_{\text {eff }}$ and $\log g$ determination, we estimated the stellar parameters of the components. These values and their uncertainties are listed in Table 2 . 

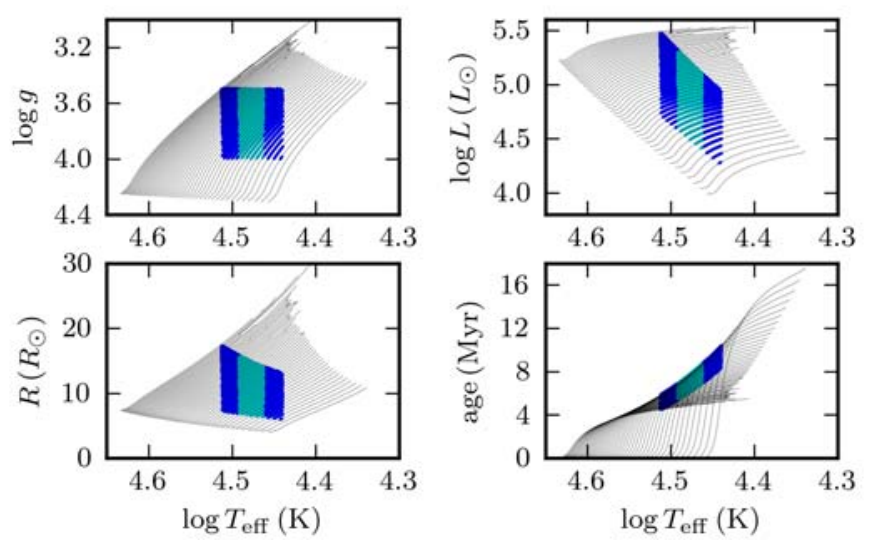

Fig. 3. Overview of the main sequence evolutionary tracks of late O-type and early B-type stars for masses of $12-35 \mathcal{M}_{\odot}$ (with a step of $0.5 \mathcal{M}_{\odot}$ ) and a core overshoot parameter $\alpha_{\text {ov }}=0.2$ pressure scale heights. Grid-points that fall within the error boxes of the primary and secondary components are shown with cyan and blue shading, respectively, on the $\log T_{\text {eff }}-\log g$ (upper left), $\log T_{\text {eff }}-\log L$ (upper right), $\log T_{\text {eff }}-R$ (lower left), and $\log T_{\text {eff }}-$ age (lower right) diagrams.

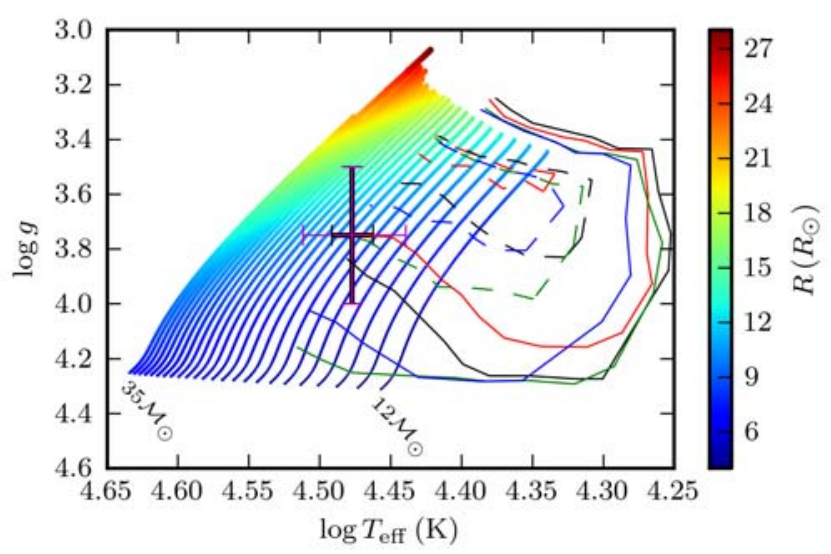

Fig. 4. $\log T_{\text {eff }}-\log g$ diagram of late O-type and early B-type stars. The spectroscopic parameters and corresponding error estimations are indicated by thick and thin crosses for the primary and secondary components, respectively. The main sequence evolutionary tracks are for masses of 12-35 $\mathcal{M}_{\odot}$ (with a step of $1 \mathcal{M}_{\odot}$ ) and a core overshoot parameter $\alpha_{\mathrm{ov}}=0.2$ pressure scale heights, and their shading and thickness indicate the corresponding radius values. Various $\beta$ Cep instability strips for $Z=0.020$ (continuous lines) and $Z=0.010$ (dashed lines), both for four different combinations of metal mixtures and opacity computations (OPAL GN93, OP GN93, OPAL AGS05+Ne, and OP AGS05+Ne) are plotted with red, blue, black, and green colours, respectively. These are taken from Miglio et al. (2007), who computed them for masses up to $18 \mathcal{M}_{\odot}$.

\subsection{Constraints on the orbit}

Using the masses from the evolutionary tracks (see Table 2), the distance values (see Sect.2.1), and the visual separation from the position measurements (Table A.1), we calculated the expected orbital period of the $\mathrm{AB}$ components of the system to see if the slow change in position angle and separation is compatible with it. Assuming the simplest case, a circular, pole-on orbit, the semi-major axis (hence the radius in this simplified case) of the orbit is $a=0.0056_{-0.0030}^{+0.0036} \mathrm{pc}$ (using a distance of $d=1.7_{-0.9}^{+1.0} \mathrm{kpc}$ representing the values mentioned in Sect. 2.1, and a separation of $\rho=0.68 \pm 0.2^{\prime \prime}$ calculated from the most recent and precise interferometric measurements between 1994 and 1997). Using

$P=2 \pi \sqrt{\frac{a^{3}}{G\left(\mathcal{M}_{\mathrm{A}}+\mathcal{M}_{\mathrm{B}}\right)}}$,

we obtain an orbital period of $P=6385_{-4808}^{+9379}$ year - with the errors covering all uncertainties. These values suggest a change of $6.9_{-4.1}^{+21.0}$ degrees in position angle during 122 years, which fits the observations (showing a change of $\approx 6.3^{\circ}$ ). Assuming an edge-on orbit, we derive an upper limit of the semi-amplitude of the radial velocity: $A_{\mathrm{RV}}=5.4_{-1.8}^{+4.7} \mathrm{~km} \mathrm{~s}^{-1}$. This, combined with the shallow profiles of the secondary due to its high projected rotational velocity and the relatively short timespan of the measurements (compared to the orbital period), explains why there was no (measurable) wavelength-shift between the lines of the two components in the spectra.

\section{The CoRoT light curve}

\subsection{Frequency analysis}

HD 51756 was observed by the CoRoT satellite during the second long run of the mission ( $\mathrm{LRa} 02)$ as part of the asteroseismology programme, while monitoring a field in the direction of the Galactic anticentre from 2454784.491822 HJD for almost 115 days (from 13 November 2008 to 8 March 2009). All flagged observations were removed from the light curve, leaving us with 278131 measurements (resulting in a duty cycle of $\approx 90 \%$ ). The decreasing trend of the CoRoT light curve has a well known instrumental origin (Auvergne et al. 2009), so it was also removed by dividing by a linear fit. Because after this step there were no clear jumps or trends visible anymore, we used the resulting dataset in our analysis.

The first visual inspection of the light curve showed a very traceable repetitive pattern over almost the full timespan of the observation, with a length of $\approx 2$ days (see Fig. 5). The shape and features of the light curve show a noteworthy resemblance to the O8.5 V star HD 46149 observed during the SRa02 short run of CoRoT and analysed in detail by Degroote et al. (2010b).

We performed an iterative prewhitening procedure (see e.g. Degroote et al. 2009) using the traditional linear Scargle periodogram (Scargle 1982). This resulted in a list of amplitudes $\left(A_{j}\right)$, frequencies $\left(f_{j}\right)$, and phases $\left(\theta_{j}\right)$, by which the light curve can be modelled via $n_{j}$ frequencies in the well-known form of

$F\left(t_{i}\right)=c+\sum_{j=1}^{n_{f}} A_{j} \sin \left[2 \pi\left(f_{j} t_{i}+\theta_{j}\right)\right]$.

We considered a peak significant if it exceeds an amplitude signal-to-noise ratio of 4 (see Breger et al. 1993). The noise level was calculated as the average amplitude - before prewhitening - in a $3 d^{-1}$ interval centred on the frequency of interest. Only 27 peaks of the Scargle periodogram (Fig. 5) met the signal-tonoise criterion, and using only the significant frequencies listed in Table A.2, we are unable to model the light curve at a satisfactory level. Even using all the 93 peaks with an SNR above three, the constructed model lacks the characteristics of the original dataset, leaving most of the non-sinusoidal sharp features in the residual light curve. The noise level in the original periodogram around $1.5-5-10 \mathrm{~d}^{-1}$ is $20.7-7.8-3.7 \mathrm{ppm}$, while it is 15.7-7.1-3.5 ppm in the periodogram of the residual light curve (which was created by subtracting a model with the parameters listed in Table A.2). 

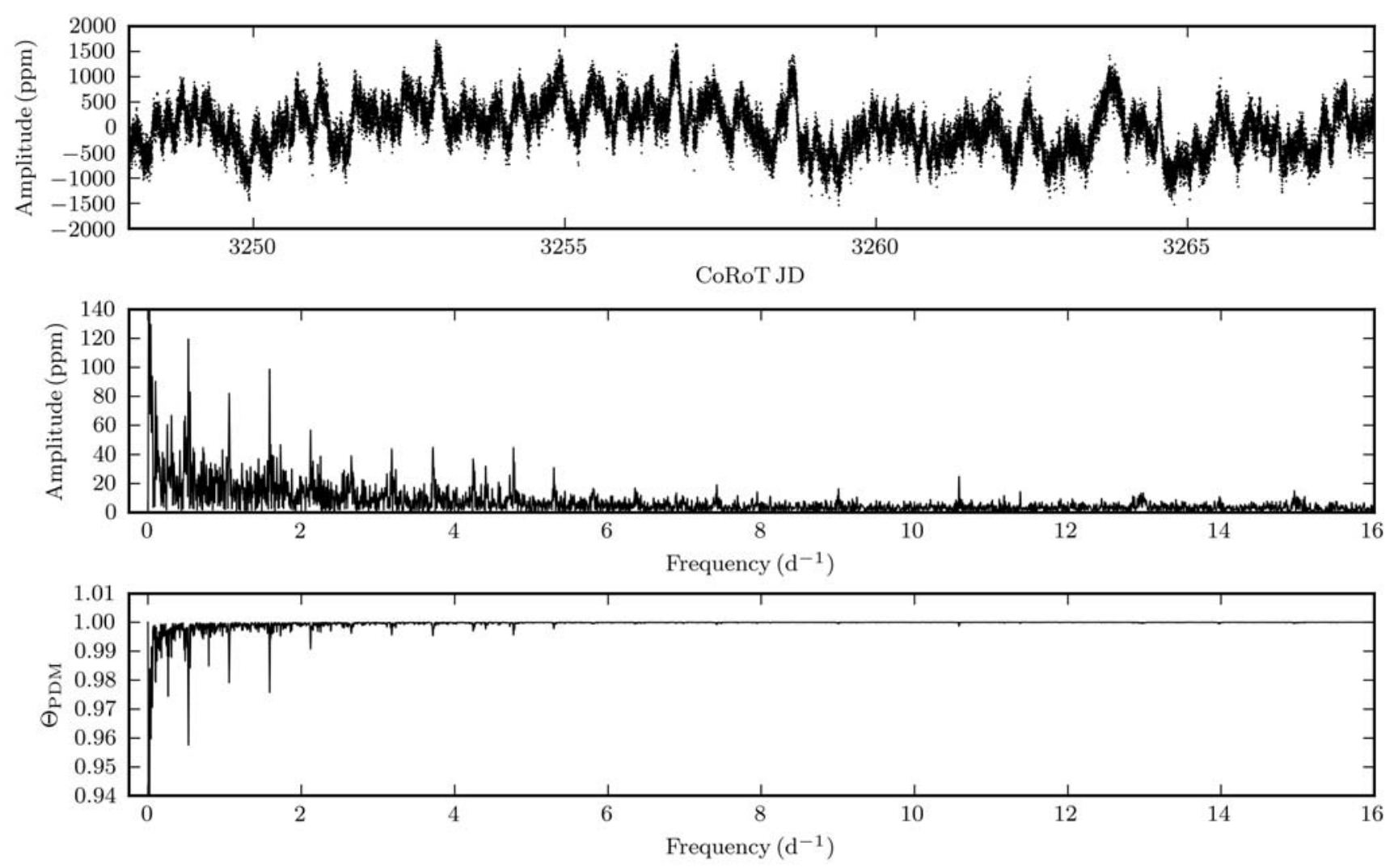

Fig. 5. (Upper panel) Part of the reduced CoRoT light curve, showing distinctive sharp recurring features every $\approx 1.9$ days. This can be seen easily around 3255 CoRoT JD, where the occurrence of features causing the highest amplitude variations is clearly periodic. (Middle panel) The Scargle periodogram of the full CoRoT light curve showing a series of harmonics at integer multiple frequencies of the main peak at $f_{0}=$ $0.52687 \pm 0.00004 \mathrm{~d}^{-1}$. (Lower panel) $\Theta_{\mathrm{PDM}}$-statistics of the light curve, showing the same structure as the Scargle periodogram.

For comparison - and because of the non-sinusoidal shape of the signal - we also used the phase dispersion minimisation (PDM) method of Stellingwerf (1978) to calculate the $\Theta_{\mathrm{PDM}^{-}}$ statistics, which shows the same global structure and features as the Scargle periodogram (see Fig. 5).

To check the stability of features in the frequency spectrum, time-resolved Fourier transformations were also calculated with different window widths. It is clearly visible from Fig. 6 that the amplitudes connected to the significant frequencies vary significantly over the span of the time series.

Of the 27 frequencies with $S N R \geq 4,11$ are connected to $f_{0}$ (Table A.2). All these frequency properties are atypical of stellar oscillations in hot B stars and point towards another origin of the variability.

\subsection{Repetitive patterns}

The classical Fourier-techniques revealed a series of harmonics at multiples of the main peak at $f_{0}=0.52687 \pm 0.00004 \mathrm{~d}^{-1}$. All harmonics (using a notation of $f_{0}^{n}$ for the $n$th harmonic of $f_{0}$ ) can be traced in the power spectrum down to $f_{0}^{15}$ (with $S N R \geq 3$ ), and $f_{0}^{17}$ and $f_{0}^{20}$ are also visible. There is a small but systematic difference between the observed $f_{0}^{n}$ and the exact $n f_{0}(n \in \mathbb{Z})$ values. Fixing the frequency values of the harmonics to $n f_{0}$ and carrying out a nonlinear least-squares fitting procedure with these peaks gives a worse model, with remaining higher residual values caused by un-prewhitened peaks right next to the removed fixed frequency harmonics. The observed frequency values are

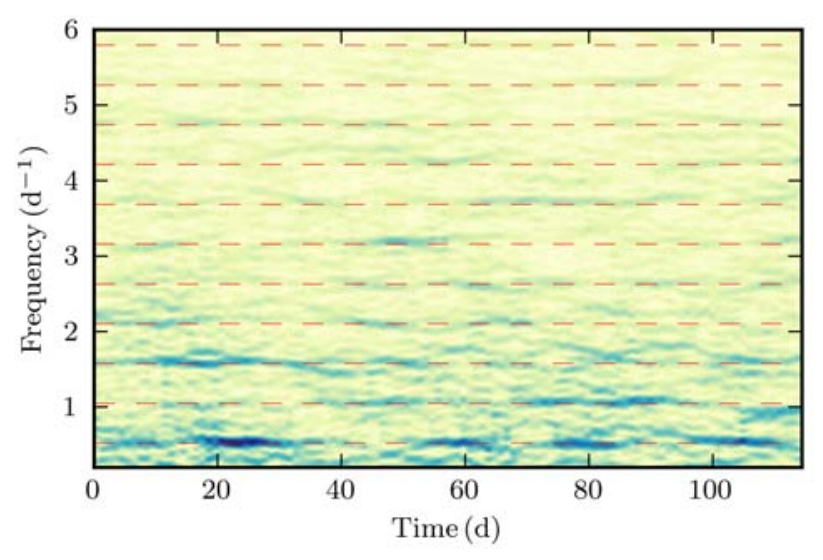

Fig. 6. Short-time Fourier transformation (window $=5 f_{0}$ ) of the $[0.2,6.0] \mathrm{d}^{-1}$ region of the light curve, showing that the amplitudes connected to different harmonics of the main peak at $f_{0}=0.52687 \pm$ $0.00004 \mathrm{~d}^{-1}$ (indicated as red dashed lines) vary significantly over time.

always higher than the ones used in the fitting (fixed at integer multiples of $f_{0}$ ), and this difference increases with frequency (or $n$ ), starting with $\Delta f_{0}^{2}=2 f_{0}-f_{0}^{2} \approx-0.008 \mathrm{~d}^{-1}$ and continuously growing to $\Delta f_{0}^{20}=20 f_{0}-f_{0}^{20} \approx-0.05 \mathrm{~d}^{-1}$. This might be a sign of rotational modulation with the rotational period of one of the components. If differential rotation were present, it could complicate the structure of the peaks and it might explain in part 

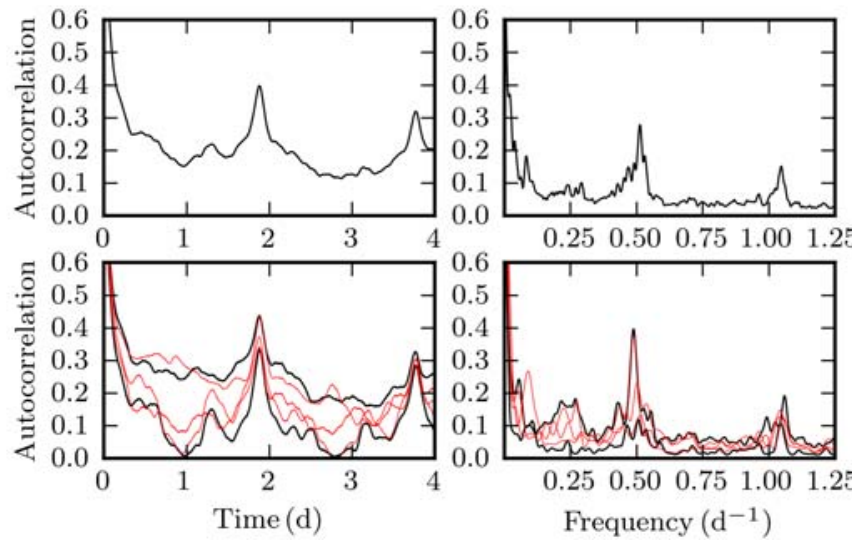

Fig. 7. Autocorrelation function of the CoRoT light curve (upper left), of the power spectrum (upper right), of the light curve cut into two and three pieces (lower left), and of the power spectra which were calculated from the pieces (two and three, plotted with thicker and thinner lines, respectively) of the light curve (lower right).

the differences between the observed peak values and exact multiples of $f_{0}$.

Another approach to searching for spacings and recurrent features both in the light curve and the power spectrum is to use the autocorrelation function. We calculated the autocorrelation of the light curve and the power spectrum (here the square of the Scargle periodogram). These tests were repeated after the dataset was cut into two, respectively three, parts. The results displayed in Fig. 7 show clear signs corresponding to the repetitive nature of the variations in the light curve. This signature stays quite clear even for shorter subsets of the data, but gets less significant in the case of the power spectrum calculated from these data sets. Furthermore, the location of the first peak of the autocorrelation function stays almost perfectly the same even for three subsets, but this stability cannot be seen for the autocorrelation of the power spectra of the subsets.

\subsection{The origin of repetitive variations}

A possible source of the variability might be atmospheric features, such as spots or chemical inhomogeneities on the surface. In those cases the observed brightness variations can be explained by the appearance and disappearance of such regions, either because of repeated creation or destruction or because of the rotation of the star. Here, we are in favour of the second option, because of the signal's self-similarity and because - as we show below - it is consistent with the determined $v \sin i$ value. For more details on how these features can actually produce the observed cusp-like peaks, we refer to Degroote (2010).

Knowing the projected rotational velocities of both components, we test that it is physically possible that one of the components has a rotational period compatible with our hypothesis that the series of harmonics in the frequency spectrum is a sign of rotational modulation. To carry out this test, we calculated the inclination values that correspond to a rotational period of $1 / f_{0}(1.898 \mathrm{~d})$ within the error bars of the $R$ and $v \sin i$ values (from Table 2), and we checked that the equatorial rotational speed at this set-up was below the critical velocity for the given $R, \mathcal{M}$ combination. If we suppose that the primary is responsible for the repetitive features in the light curve, then its inclination has to be $i_{\mathrm{A}}=6.3_{-3.0}^{+4.7}$ degrees (Fig. 8). On the other hand, if we assume that the variations are connected to the secondary, then we conclude an $i_{\mathrm{B}}=41.6_{-21.7}^{+48.4}$ degrees, and we end up with an equator-on solution already slightly before reaching the lowest possible $R$ value. Taking only the most probable $R$ values into account, we end up with $i_{\mathrm{A}}=6.3_{-0.9}^{+0.9}$ degrees and $i_{\mathrm{B}}=41.6_{-4.4}^{+4.7}$ degrees.

As is clearly visible in Fig. 8, these setups may result in exceeding the break-up velocity if the radius of the given component is in the very upper part of the error box. If the mass is lowered, the radius limit will also drop. We can conclude that most of the possible configurations are indeed compatible with our hypothesis of a rotational origin of the variability.

\subsection{Inferences from the non-detection of pulsation}

For all the models passing through the error boxes of the primary and secondary indicated in Fig. 3, we considered the frequencies of the fundamental radial mode and of the few lowest radial overtones, as well as the lowest-order $\mathrm{p}$ and $\mathrm{g}$ axisymmetric modes of degrees 1 and 2. None of those eigenfrequencies come close to $f_{0}$. It is thus very unlikely that $f_{0}$, with so many harmonics present in the light curve, results from a pulsation mode, as one does not expect a high-overtone mode or a high-degree mode to show so many harmonics of its frequency (e.g., Degroote et al. 2009).

We made non-adiabatic computations (with the code MAD developed by Dupret et al. 2002) for these models to check which modes are predicted to be excited by the present input physics and theory of mode excitation (see Fig. 9). We need to understand why the star does not oscillate, as the theoretical calculations predict $\mathrm{p}$ modes to be excited throughout the error box of HD 51756, except the very bottom left region. Typical frequencies are $3.5-6.5 \mathrm{~d}^{-1}$ around the center of our error box. This range extends to $1.9 \mathrm{~d}^{-1}$ and $9.4 \mathrm{~d}^{-1}$ towards the lower and higher gravity values, respectively. Moreover, g modes are also expected to be excited in some of the higher mass models, with frequencies near $0.1 \mathrm{~d}^{-1}$.

Finally, we should remember that CoRoT measured the integrated flux from two stars, therefore the observed amplitude of the rotational modulation is damped. This would also be true for the amplitudes of the pulsational modes, if any.

\section{Conclusions}

We analysed the high-resolution spectroscopy and high-quality space-based photometry of HD 51756. The former - via synthetic spectrum fitting and matching the results with evolutionary tracks - enabled us to derive the fundamental stellar parameters of both components of the underlying double-lined spectroscopic binary, while the latter led us to conclude that none of these stars show pulsations. We interpret the repetitive patterns of the light curve, the observed harmonic structure in the frequency spectrum, and the strong, stable peaks in the autocorrelation functions as signs of rotational modulation. This hypothesis is compatible with the observed and deduced stellar parameters.

The absence of oscillations in the components of HD 51756 requires an explanation. It has recently been found by Balona et al. (in prep.) that some stars observed with the Kepler space mission, which are also monitored at $\mu$ mag precision and situated in the $\beta$ Cep instability strip, do not pulsate either. A mixture of non-pulsators and pulsators with or without rotational modulation has already been established from ground-based data for stars in the g-mode instability strip of the cooler slowly 

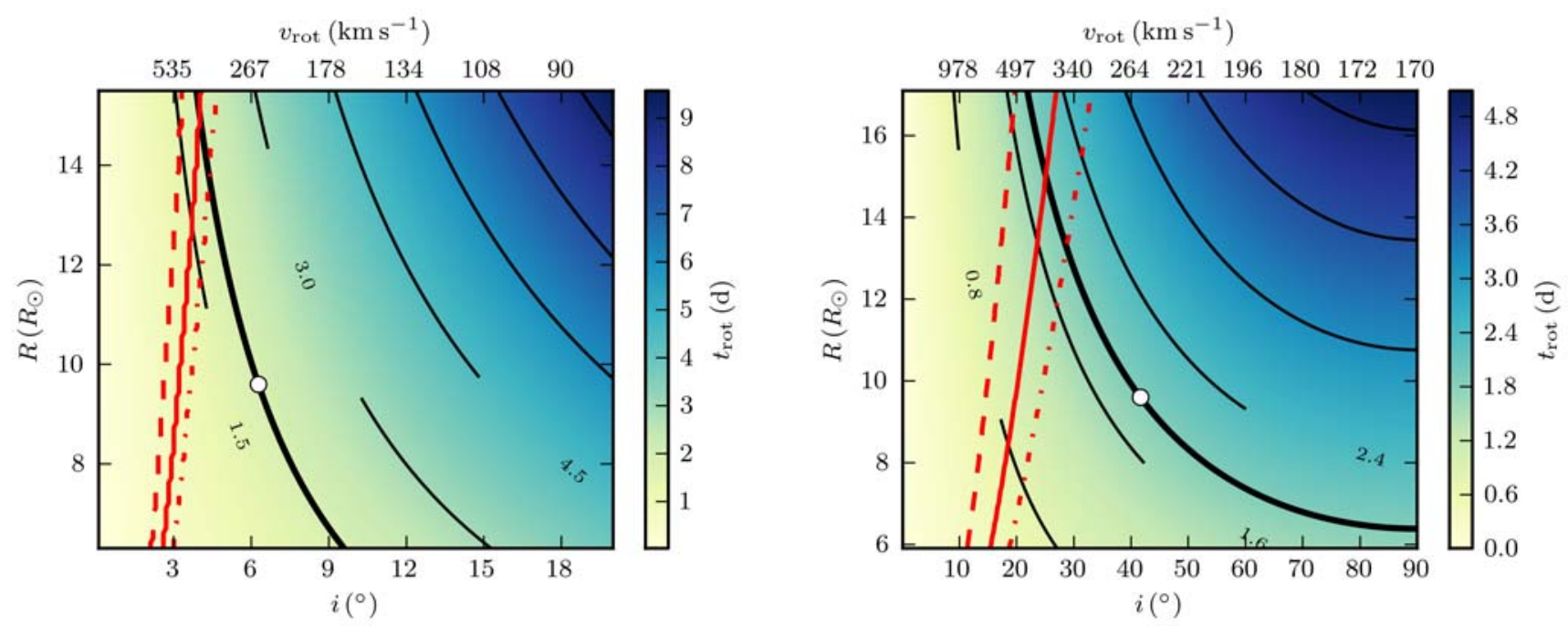

Fig. 8. Possible $i, R, v_{\text {rot }}$ combinations in agreement with the observed $v \sin i=28 \mathrm{~km} \mathrm{~s}^{-1}$ of the primary (left) and the $v \sin i=170 \mathrm{~km} \mathrm{~s}{ }^{-1}$ of the secondary component (right). Plots show (via colours and thin black curved contour lines) rotation periods calculated from the observed projected rotational velocities at given $i, R$ values. The thick contour line corresponds to the main peak at $1.898 \mathrm{~d}$ from the frequency spectrum, and the dashed, continuous, and dot-dashed red lines show the break-up velocity (with speeds rising towards the left) for $\mathcal{M}_{\max }$, for $\mathcal{M}_{\text {, and for }} \mathcal{M}_{\text {min }}$, respectively. The thick contour line corresponds with the parameter-combinations that fit the hypothesis in Sect. 3.3, and the white dot shows the location of the most probable $R$ (and thus $i$ ) value from Table 2 .
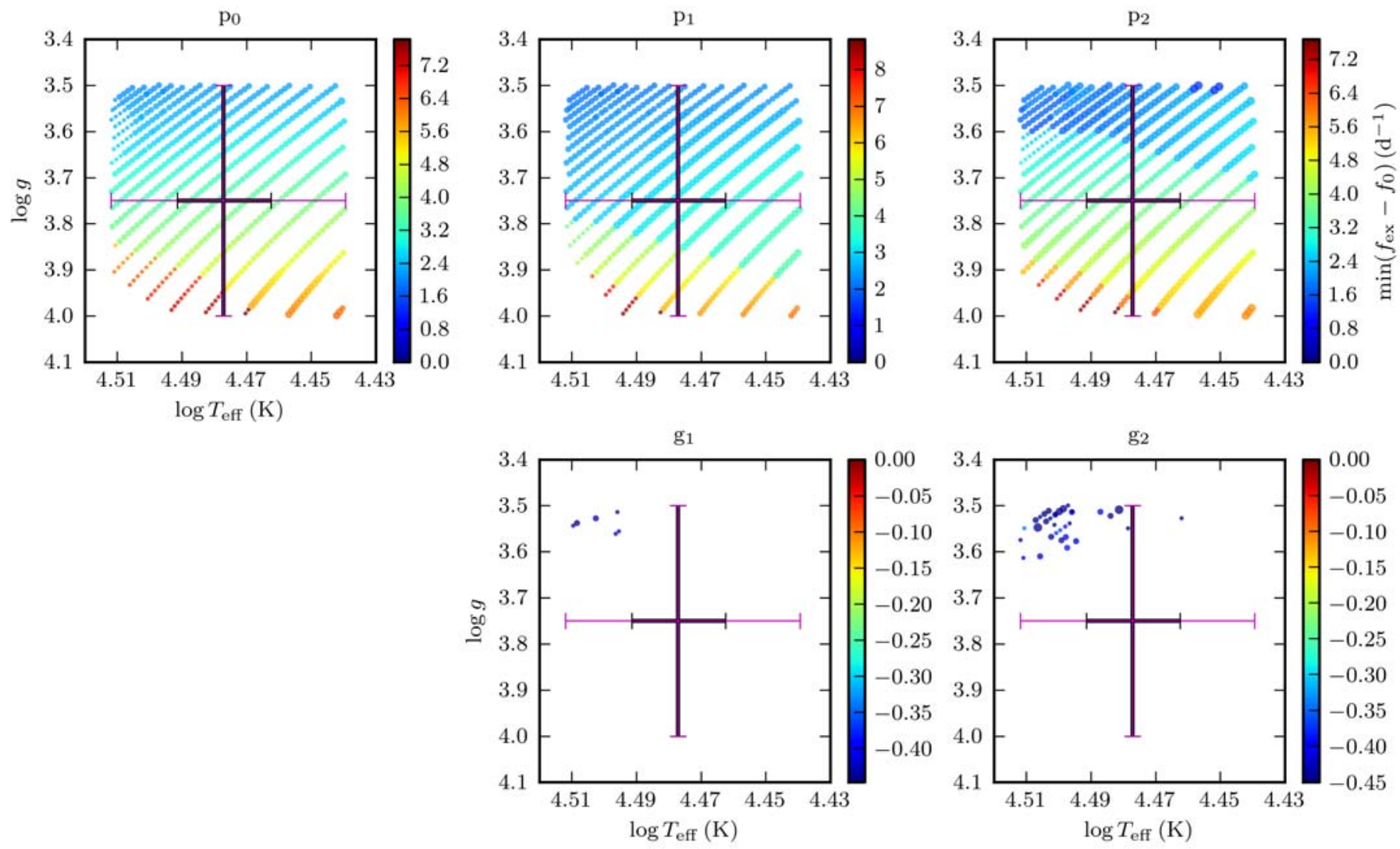

Fig. 9. $\log T_{\text {eff }}-\log g$ diagrams showing an overview of models where different modes are predicted to be excited in our non-adiabatic computations for the error box of the components of HD 51756. Each filled circle represents a model along the evolutionary tracks where modes are predicted to be excited. The size of the symbols refers to the number of excited modes (1-4 per mode type), while the colour corresponds to the frequency difference between $f_{0}$ and the closest mode in the model. The spectroscopic parameters and corresponding error estimations of HD 51756 are indicated by thick and thin crosses for the primary and secondary component, respectively. 
pulsating B stars (e.g., Briquet et al. 2004). It was interpreted as due to a difference in magnetic field strength and age, since the $\mathrm{Bp}$ stars are younger and have stronger fields than the pulsators, although this interpretation is still a matter of debate (e.g., Briquet et al. 2007a; Silvester et al. 2009; Hubrig et al. 2011). As far as we are aware, no magnetic field measurements are available for HD 51756.

Acknowledgements. The research leading to these results has received funding from the European Research Council under the European Community's Seventh Framework Programme (FP7/2007-2013)/ERC grant agreement No. 227224 (PROSPERITY), as well as from the Research Council of K.U. Leuven grant agreement GOA/2008/04. E.N. acknowledges financial support of the NN203 302635 grant from the MNiSW. E.P. and M.R. acknowledge financial support from the Italian ESS project, contract ASI/INAF I/015/07/0, WP 03170. This research has made use of the Washington Double Star Catalog maintained at the US Naval Observatory.

\section{References}

Abt, H. A. 2008, ApJS, 176, 216

Aerts, C., Christensen-Dalsgaard, J., \& Kurtz, D. W. 2010, Asteroseismology (Springer)

Aerts, C., Thoul, A., Daszyńska, J., et al. 2003, Science, 300, 1926

Ausseloos, M., Scuflaire, R., Thoul, A., \& Aerts, C. 2004, MNRAS, 355, 352

Auvergne, M., Bodin, P., Boisnard, L., et al. 2009, A\&A, 506, 411

Avedisova, V. S., \& Kondratenko, G. I. 1984, Nauchnye Informatsii, 56, 59

Baade, D., Daachs, J., van de Weygaert, R., \& Steeman, F. 1988, A\&A, 198, 211

Baglin, A., Auvergne, M., Barge, P., et al. 2006, in ESA Special Publication, ed.

M. Fridlund, A. Baglin, J. Lochard, \& L. Conroy, 1306, 33

Baize, P. 1938, J. Obs., 21, 161

Baranne, A., Queloz, D., Mayor, M., et al. 1996, A\&AS, 119, 373

Behall, A. L. 1976, Publications of the US Naval Observatory Second Series, 24,1

Belkacem, K., Samadi, R., Goupil, M., et al. 2009, Science, 324, 1540

Breger, M., Stich, J., Garrido, R., et al. 1993, A\&A, 271, 482

Briquet, M., Aerts, C., Lüftinger, T., et al. 2004, A\&A, 413, 273

Briquet, M., Hubrig, S., De Cat, P., et al. 2007a, A\&A, 466, 269

Briquet, M., Morel, T., Thoul, A., et al. 2007b, MNRAS, 381, 1482

Briquet, M., Uytterhoeven, K., Morel, T., et al. 2009, A\&A, 506, 269

Briquet, M., Aerts, C., Baglin, A., et al. 2011, A\&A, 527, A112

Burnham, S. W. 1875, AN, 86, 337

Degroote, P. 2010, PhD Thesis, K. U. Leuven

Degroote, P., Briquet, M., Catala, C., et al. 2009, A\&A, 506, 111

Degroote, P., Aerts, C., Baglin, A., et al. 2010a, Nature, 464, 259

Degroote, P., Briquet, M., Auvergne, M., et al. 2010b, A\&A, 519, A38

Desmet, M., Briquet, M., Thoul, A., et al. 2009, MNRAS, 396, 1460

Donati, J., Semel, M., Carter, B. D., Rees, D. E., \& Collier Cameron, A. 1997, MNRAS, 291, 658

Doolittle, E. 1905, Publications of the Flower Astronomical Observatory, 2, 1

Douglass, G. G., Mason, B. D., Germain, M. E., \& Worley, C. E. 1999, AJ, 118, 1395

Dupret, M., De Ridder, J., Neuforge, C., Aerts, C., \& Scuflaire, R. 2002, A\&A, 385,563

Dworetsky, M. M., Whitelock, P. A., \& Carnochan, D. J. 1982, MNRAS, 200, 445

Eddington, S., Bryant, W., Chapman, S., et al. 1912, MNRAS, 73, 93

ESA 1997, VizieR Online Data Catalog, 1239, 0
Fabricius, C., Høg, E., Makarov, V. V., et al. 2002, A\&A, 384, 180

Fich, M., \& Blitz, L. 1984, ApJ, 279, 125

Floquet, M., Hubert, A., Huat, A., et al. 2009, A\&A, 506, 103

Germain, M. E., Douglass, G. G., \& Worley, C. E. 1999, AJ, 117, 1905

Glebocki, R., \& Stawikowski, A. 2000, Acta Astron., 50, 509

Gulati, R. K., Malagnini, M. L., \& Morossi, C. 1989, A\&AS, 80, 73

Gutiérrez-Soto, J., Floquet, M., Samadi, R., et al. 2009, A\&A, 506, 133

Handler, G. 2009, MNRAS, 398, 1339

Handler, G., Jerzykiewicz, M., Rodríguez, E., et al. 2006, MNRAS, 365, 327

Hill, G. 1967, ApJS, 14, 263

Horch, E., van Altena, W. F., Girard, T. M., et al. 2001, AJ, 121, 1597

Huat, A., Hubert, A., Baudin, F., et al. 2009, A\&A, 506, 95

Hubrig, S., Ilyin, I., Schöller, M., et al. 2011, ApJ, 726, L5

Kaltcheva, N. T., \& Hilditch, R. W. 2000, MNRAS, 312, 753

Kaufer, A., Stahl, O., Tubbesing, S., et al. 1999, The Messenger, 95, 8

Lanz, T., \& Hubeny, I. 2003, ApJS, 146, 417

Lanz, T., \& Hubeny, I. 2007, ApJS, 169, 83

Leavenworth, F., \& Beal, W. O. 1930, Measures of Double Stars, ed. F. Leavenworth, \& W. O. Beal

Lewis, T., Bowyer, W., Bryant, W., \& Furner, H. 1904, MNRAS, 64, 789

Mayor, M., Pepe, F., Queloz, D., et al. 2003, The Messenger, 114, 20

Mazumdar, A., Briquet, M., Desmet, M., \& Aerts, C. 2006, A\&A, 459, 589

Michel, E., Baglin, A., Auvergne, M., et al. 2006, in ESA SP, ed. M. Fridlund, A. Baglin, J. Lochard, \& L. Conroy, 1306, 39

Miglio, A., Montalbán, J., \& Dupret, M. 2007, CoAst, 151, 48

Morgan, W. W., Code, A. D., \& Whitford, A. E. 1955, ApJS, 2, 41

Münch, L. 1951, ApJ, 113, 309

Neiner, C., Gutiérrez-Soto, J., Baudin, F., et al. 2009, A\&A, 506, 143

Olivier, C. P. 1907, AN, 174, 209

Olivier, C. P. 1928 , AN, 233, 393

Olivier, C. P. 1932, Publications of the Flower Astronomical Observatory, 5, 1

Olivier, C. P., Wilson, R. E., \& Neff, W. N. 1909, AN, 182, 253

Pamyatnykh, A. A., Handler, G., \& Dziembowski, W. A. 2004, MNRAS, 350, 1022

Perryman, M. A. C., Lindegren, L., Kovalevsky, J., et al. 1997, A\&A, 323, L49

Przybilla, N., Nieva, M., \& Butler, K. 2008, ApJ, 688, L103

Queloz, D., Mayor, M., Udry, S., et al. 2001, The Messenger, 105, 1

Rivinius, T., Baade, D., Štefl, S., et al. 2001, A\&A, 369, 1058

Rivinius, T., Baade, D., \& Štefl, S. 2003, A\&A, 411, 229

Savage, B. D., Massa, D., Meade, M., \& Wesselius, P. R. 1985, ApJS, 59, 397

Scargle, J. D. 1982, ApJ, 263, 835

Silvester, J., Neiner, C., Henrichs, H. F., et al. 2009, MNRAS, 398, 1505

Solano, E., Catala, C., Garrido, R., et al. 2005, AJ, 129, 547

Stellingwerf, R. F. 1978, ApJ, 224, 953

Stone, O. 1879, Publications of the Cincinnati Observatory, 5, 1

Tarrant, K. J. 1890, AN, 125, 225

van Leeuwen, F. 2007, A\&A, 474, 653

Vogt, N. 1976, A\&A, 53, 9

Voûte, J. 1955, J. Obs., 38, 109

Walker, R. L. 1966, Publications of the US Naval Observatory Second Series, 18,1

Walker, Jr., R. L. 1969, Publications of the US Naval Observatory Second Series, 22,1

Weber, S. V., Henry, R. C., \& Carruthers, G. R. 1971, ApJ, 166, 543

Wilson, R. E. 1953, Carnegie Institute Washington D.C. Publication, 0

Worley, C. E. 1957, AJ, 62, 153

Worley, C. E. 1963 , AJ, 68, 114

Worley, C. E. 1972, Publications of the US Naval Observatory Second Series, 22,2

Wright, C. O., Egan, M. P., Kraemer, K. E., \& Price, S. D. 2003, AJ, 125, 359

Zima, W. 2008, CoAst, 155, 17 


\section{Appendix A: Tables}

Table A. 1 contains all the position measurements of HD 51756 (catalogued as BU 327, but widely known as WDS J065850301) found in the Washington Double Star Catalog. Error estimates - when available - can be found in the original references.

Table A.2 lists the Fourier parameters of significant peaks in the periodogram.
Table A.1. Position measurements of HD 51756 AB.

\begin{tabular}{|c|c|c|c|c|c|}
\hline Date & $\theta\left(^{\circ}\right)$ & $\rho\left(^{\prime \prime}\right)$ & $N$ & Method $^{a}$ & Reference $^{b}$ \\
\hline 1875.06 & 90 & 1 & 1 & $\mathrm{~A}$ & 1 \\
\hline 1876.83 & 100.8 & 0.96 & 2 & A & D1883 \\
\hline 1878.12 & 97.2 & $\mathrm{n} / \mathrm{a}$ & $\overline{1}$ & A & 2 \\
\hline 1881.05 & 96.1 & 0.75 & 1 & A & Bu1883 \\
\hline 1888.10 & 95.8 & 0.73 & 2 & A & 3 \\
\hline 1888.222 & 95.5 & 0.84 & 4 & B & 4 \\
\hline 1892.08 & 95.8 & 0.79 & 3 & $\mathrm{~A}$ & Bu1894 \\
\hline 1894.40 & 98.1 & 0.64 & 5 & A & Sp1909 \\
\hline 1899.01 & 93.6 & 0.59 & 1 & A & Bu1900 \\
\hline 1902.544 & 93.9 & 0.68 & 6 & A & 5 \\
\hline 1903.14 & 100.0 & 0.64 & 2 & A & 9 \\
\hline 1906.16 & 95.9 & 0.87 & 3 & A & 6 \\
\hline 1906.17 & 101.5 & 0.81 & 1 & A & Frm1907 \\
\hline 1909.06 & 100.4 & 0.90 & 1 & A & 7 \\
\hline 1909.06 & 101.3 & 0.88 & 1 & A & 7 \\
\hline 1909.13 & 96.9 & 0.70 & 2 & A & Wz1912 \\
\hline 1911.15 & 91.7 & 0.56 & 2 & A & 8 \\
\hline 1920.09 & 98.1 & 0.77 & 1 & A & 10 \\
\hline 1924.35 & 99.4 & 0.83 & 3 & A & 10 \\
\hline 1924.79 & 97.5 & 0.94 & 1 & A & 10 \\
\hline 1930.57 & 98.3 & 0.71 & 2 & A & Bon1938 \\
\hline 1930.84 & 96.2 & 0.80 & 3 & A & 11 \\
\hline 1935.43 & 102.0 & 0.78 & 4 & A & 12 \\
\hline 1942.88 & 95.1 & 0.62 & 3 & A & 13 \\
\hline 1945.31 & 103.6 & 0.68 & 2 & $\mathrm{~B}$ & VBs 1954 \\
\hline 1947.17 & 102.8 & 0.76 & 2 & $\mathrm{~A}$ & VBs 1954 \\
\hline 1953.22 & 98.5 & 0.81 & 6 & A & Rab1961b \\
\hline 1956.11 & 98.2 & 0.74 & 2 & $\mathrm{~A}$ & 14 \\
\hline 1961.04 & 101.6 & 0.67 & 4 & A & 15 \\
\hline 1965.034 & 100.1 & 0.69 & 4 & A & 16 \\
\hline 1966.182 & 101.4 & 0.58 & 3 & $\mathrm{~A}$ & 17 \\
\hline 1968.045 & 101.4 & 0.66 & 3 & A & 18 \\
\hline 1974.717 & 101.1 & 0.59 & 3 & $\mathrm{~A}$ & $19^{c}$ \\
\hline 1975.522 & 101.6 & 0.55 & 3 & B & Wak1985 \\
\hline 1991.25 & 102. & 0.71 & 1 & $\mathrm{~T}$ & 20 \\
\hline 1991.92 & 104.0 & 0.68 & 1 & $\mathrm{~T}$ & 21 \\
\hline 1994.129 & 101.2 & 0.67 & 1 & S & 22 \\
\hline 1994.194 & 99.7 & 0.63 & 1 & S & 22 \\
\hline 1994.194 & 100.5 & 0.68 & 1 & $\mathrm{~S}$ & 22 \\
\hline 1995.188 & 100.6 & 0.66 & 1 & $\mathrm{~S}$ & 22 \\
\hline 1995.199 & 103.0 & 0.68 & 1 & $\mathrm{~S}$ & 22 \\
\hline 1995.202 & 102.1 & 0.70 & 1 & $\mathrm{~S}$ & 22 \\
\hline 1995.202 & 101.8 & 0.67 & 1 & S & 22 \\
\hline 1995.205 & 101.5 & 0.69 & 1 & S & 22 \\
\hline 1995.207 & 101.9 & 0.63 & 1 & S & 22 \\
\hline 1996.8960 & 101.5 & 0.695 & 1 & $\mathrm{~S}$ & 23 \\
\hline 1997.184 & 101.5 & 0.67 & 1 & S & 24 \\
\hline 1997.184 & 102.2 & 0.69 & 1 & $\mathrm{~S}$ & 24 \\
\hline
\end{tabular}

Notes. (a) Methods of observations. A: Refractor, micrometer; B: Reflector, micrometer; T: Hipparcos or Tycho observation; S: Speckle interferometry. (b) For special references - which are not in the SAO/NASA Astrophysics Data System - see the References and discoverer codes of The Washington Double Star Catalog at http://ad. usno.navy.mil/wds/Webtextfiles/wdsnewref.txt . (c) These observations were made after the closing of the cited article, but before the retirement of Alan Behall. Observing techniques, instrumentation and particulars are the same as described by Behall (1976) .

References. (1) Burnham (1875); (2) Stone (1879); (3) Leavenworth \& Beal (1930); (4) Tarrant (1890); (5) Doolittle (1905); (6) Olivier (1907); (7) Olivier et al. (1909); (8) Eddington et al. (1912); (9) Lewis et al. (1904); (10) Olivier (1928); (11) Olivier (1932); (12) Baize (1938); (13) Voûte (1955); (14) Worley (1957); (15) Worley (1963); (16) Walker (1966); (17) Walker (1969); (18) Worley (1972); (19) Behall (1976); (20) ESA (1997); (21) Fabricius et al. (2002); (22) Germain et al. (1999); (23) Horch et al. (2001); (24) Douglass et al. (1999). 
Table A.2. Table of Fourier parameters (frequencies $\left(f_{j}\right)$, amplitudes $\left(A_{j}\right)$, and phases $\left(\theta_{j}\right)$ ) of peaks with a signal-to-noise ratio $(S N R) \geq 4$, plus clear harmonics $\left(f_{0}^{n}\right)$ of the main peak at $f_{0}=0.52687 \pm 0.00004 \mathrm{~d}^{-1}$ down to $S N R \geq 3$. SNR is calculated over $3 \mathrm{~d}^{-1}$ in the periodogram.

\begin{tabular}{|c|c|c|c|c|c|c|c|}
\hline$f\left(\mathrm{~d}^{-1}\right)$ & $\epsilon_{f}\left(\mathrm{~d}^{-1}\right)$ & $A(\mathrm{ppm})$ & $\epsilon_{A}(\mathrm{ppm})$ & $\theta(2 \pi / \mathrm{rad})$ & $\epsilon_{\theta}(2 \pi / \mathrm{rad})$ & SNR & note \\
\hline 0.009071 & 0.000035 & 167.4 & 1.1 & 0.2563 & 0.0073 & 10.4 & \\
\hline 0.017911 & 0.000030 & 183.8 & 1.1 & -0.1304 & 0.0063 & 12.2 & \\
\hline 0.033302 & 0.000042 & 137.5 & 1.1 & -0.0228 & 0.0087 & 8.5 & \\
\hline 0.040540 & 0.000060 & 80.1 & 1.0 & 0.3908 & 0.0124 & 6.0 & \\
\hline 0.062041 & 0.000057 & 89.8 & 1.0 & 0.2046 & 0.0118 & 6.4 & \\
\hline 0.098717 & 0.000058 & 102.2 & 1.0 & -0.0143 & 0.0121 & 6.2 & \\
\hline 0.119596 & 0.000080 & 58.2 & 0.9 & -0.0415 & 0.0165 & 4.5 & \\
\hline 0.137555 & 0.000083 & 59.1 & 0.9 & 0.4332 & 0.0173 & 4.3 & \\
\hline 0.253269 & 0.000075 & 60.2 & 0.9 & 0.3487 & 0.0156 & 4.7 & \\
\hline 0.306826 & 0.000071 & 63.9 & 1.0 & -0.3190 & 0.0146 & 5.1 & \\
\hline 0.472920 & 0.000071 & 65.3 & 1.0 & -0.2757 & 0.0147 & 5.0 & \\
\hline 0.485587 & 0.000081 & 69.5 & 0.9 & -0.2933 & 0.0168 & 4.4 & \\
\hline 0.496949 & 0.000088 & 47.8 & 0.9 & 0.1944 & 0.0182 & 4.1 & \\
\hline 0.526865 & 0.000042 & 124.1 & 1.0 & -0.3897 & 0.0088 & 8.5 & $f_{0}$ \\
\hline 0.537154 & 0.000075 & 61.5 & 0.9 & -0.0783 & 0.0155 & 4.8 & \\
\hline 0.551246 & 0.000060 & 69.7 & 1.0 & 0.0598 & 0.0124 & 6.0 & \\
\hline 1.061396 & 0.000058 & 94.0 & 1.0 & -0.3826 & 0.0120 & 6.2 & $f_{0}^{2}$ \\
\hline 1.588351 & 0.000050 & 101.1 & 1.0 & 0.3509 & 0.0104 & 7.2 & $f_{0}^{3}$ \\
\hline 2.122824 & 0.000081 & 58.3 & 0.9 & -0.3705 & 0.0167 & 4.4 & $f_{0}^{4}$ \\
\hline 2.653912 & 0.000106 & 39.1 & 0.9 & -0.0592 & 0.0219 & 3.4 & $f_{0}^{5}$ \\
\hline 3.181300 & 0.000098 & 42.5 & 0.9 & 0.2905 & 0.0203 & 3.8 & $f_{0}^{6}$ \\
\hline 3.718828 & 0.000094 & 45.3 & 0.9 & -0.1821 & 0.0195 & 4.3 & $f_{0}^{7}$ \\
\hline 4.243161 & 0.000110 & 37.2 & 0.8 & -0.1519 & 0.0229 & 4.1 & $f_{0}^{8}$ \\
\hline 4.769149 & 0.000094 & 45.5 & 0.9 & 0.1209 & 0.0195 & 5.5 & $f_{0}^{9}$ \\
\hline 5.297008 & 0.000119 & 31.9 & 0.8 & -0.1407 & 0.0248 & 4.6 & $f_{0}^{10}$ \\
\hline 5.809200 & 0.000188 & 17.3 & 0.6 & 0.0690 & 0.0390 & 3.1 & $f_{0}^{11}$ \\
\hline 6.354499 & 0.000185 & 16.4 & 0.6 & -0.3715 & 0.0384 & 3.2 & $f_{0}^{12}$ \\
\hline 6.894291 & 0.000213 & 13.5 & 0.6 & 0.4758 & 0.0442 & 3.1 & $f_{0}^{13}$ \\
\hline 7.421695 & 0.000163 & 19.8 & 0.7 & -0.2722 & 0.0338 & 4.2 & $f_{0}^{14}$ \\
\hline 7.949167 & 0.000198 & 14.9 & 0.6 & -0.1871 & 0.0411 & 3.6 & $f_{0}^{15}$ \\
\hline 9.009843 & 0.000178 & 17.3 & 0.6 & -0.4902 & 0.0369 & 4.6 & $f_{0}^{17}$ \\
\hline 10.583197 & 0.000140 & 24.9 & 0.7 & -0.3271 & 0.0291 & 7.4 & $f_{0}^{20}$ \\
\hline 13.969336 & 0.000292 & 9.0 & 0.5 & 0.1131 & 0.0606 & 4.0 & \\
\hline
\end{tabular}

Notes. The group of low-frequency values show up because of noise and not completely removed trends. Several frequencies close to $f_{0}$ can be explained by the fact that neither the frequency nor the amplitude of $f_{0}$ is stable, as it can be clearly seen in Fig. $6 .{ }^{(a)}$ Peak corresponding to the orbital period of the CoRoT satellite. 